\title{
Quantitative Efficacy Assessment of AAV2-EPO to DR
}

\author{
H.Y. Liu, Q.L. Li, Y.F. Qian \\ Shanghai Key Laboratory of Multidimensional Information \\ Processing \\ East China Normal University \\ Shanghai, Chinac
}

\begin{abstract}
AAV2-EPO (Adeno-associated virus type 2-mediated EPO) efficacy to early diabetic retinopathy (DR) of experimental rats was studied by molecular hyperspectral imaging system (MHIS). Molecular hyperspectral images of rat retinal tissue slice were collected in the normal control group $\mathrm{N}$, the diabetic group D, the diabetes treatment group by AAV2-EPO (low dose group E1, middle dose group E2, high dose group E3). By quantitatively assessment of the thickness of the outer nuclear layer (ONL), the relative error of the spectrum, spectral similarity comparison on the spectrum angle and the correlation coefficient, it were proves that AAV2-EPO has some efficacy to early diabetic retinopathy of rats. AAV2-EPO treatment with middle dose can be most effective in reducing nerve cells apoptosis of retinal outer nuclear layer.
\end{abstract}

Keywords-diabetic retinopathy; MHSI; AAV2-EPO; ONL; qualitative analysis; spectral angle

\section{INTRODUCTION}

Diabetes-induced diabetic retinopathy (DR) is a serious complication so that it becomes a major cause of blindness to global population [1]. During pathophysiological changes of DR, optic nerve apoptosis is an important part [2]. In recent years some papers have reported it can effectively protect retinal neurons after intravitreal injection of AAV2-EPO, so as to achieve the early experimental prevention and treatment to DR [3-4].

By using virus mediated EPO protein with AAV2, AAV2 EPO was given only once, then the virus in the cells within the eyes will be integrated. And EPO proteins were expressed continuously and play a protective role [5-6]. This operation is easy to be accepted by the patients in clinical application.

Self-developed molecular hyperspectral imaging system (MHIS) was used in the observation of rat retinal tissue sections. Three dimensional image-spectrum data are provided, which assisted the researchers to study disease pathogenesis of DR and quantitatively assess efficacy of AAV2-EPO to DR.

\section{MATERIALS AND METHODS}

\section{A. Samples and Preparation}

Select 40 healthy adult male Wistar rats of individual quality about $230 \sim 280 \mathrm{~g}$. Refractive mediua of selected rats is clear and round pupil has a good react to light and fundus has no abnormal disease. Diabetic rat model were established by injection of STZ (Streptozotocin). From 40 healthy rats 8 rats were randomly selected as a normal control group (Code $\mathrm{N}$ ).

\author{
J.F. Zhang \\ Department of Regenerative Medicine and Tongji Eye Institute \\ Shanghai, China
}

Remaining 32 rats were induced diabetes by intraperitoneal injection STZ. After two weeks, 8 rats were randomly selected as the diabetic group (Code D) from 32 diabetes rats. The remaining 24 rats were divided into three groups and respectively used different doses of intravitreal injection of AAV2-EPO.The three groups were as the drug treatment group. The low dose treatment group was coded E1. The middle dose treatment group was coded E2. The high dose treatment group was coded E3. After 14 days feeding of $\mathrm{N}$ and $\mathrm{D}$ group rats and 45 days feeding of E1, E2, E3 group rats, they were sacrificed by general anesthesia and their eyeballs were removed. The removed eyeballs were placed in 4\% PBS (phosphate solution) buffer more than 24h. Under a microscope, the anterior segment of eyeball (including the cornea, iris and lens) was removed, and outside eye tissues was weeded out and eye cup was remained. Eye cup was put into the $10 \%, 20 \%$ and $30 \%$ PBS buffer sucrose and achieved gradient dehydration. By sliced with frozen slicer machine rats optic nerve samples were obtained and be dyed with HE reagents.

\section{B. AOTF (Acousto-optic Tunable Filters)-based Molecular} Hyperspectral Imaging System

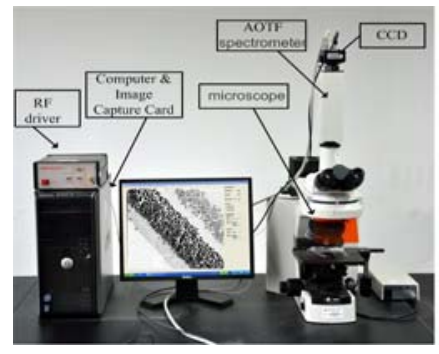

FIGURE I. MOLECULAR HYPERSPECTRAL IMAGING (MHSI) SYSTEM.

MHIS provides image information and spectral information of biological tissue samples at the same time. From information, qualitative and quantitative description and positioning of the detection target can be achieved to benefit certain early pathological diagnosis [7].

System spectral range is $550 \mathrm{~nm}$ to $1000 \mathrm{~nm}$. Effective pixels of the image are $1024 \times 1024$. The spectral resolution is better than $2 \mathrm{~nm}$. The spatial resolution is about $0.0615 \mu \mathrm{m}$. Capturing image speed is up $2.6125 \mathrm{~s} / \mathrm{B}$. 
C. Molecular Hyperspectral Image Data and Data Preprocessing

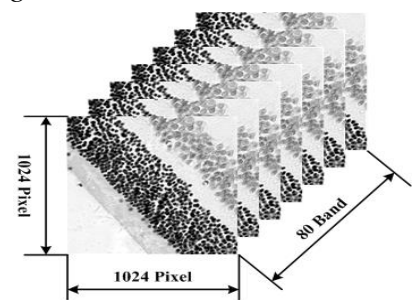

FIGURE II. MOLECULAR HYPERSPECTRAL IMAGING DATA.

System data format uses BSQ (Band Sequential), namely the band order format storage methods. In the process of sample data collection, we collect orderly images of the first band of images, the second band, and so on, until the 80th band image.See Figure 2, image spatial resolution is 1024 pixels $x$ 1024 pixels. In data storage, each pixel includes 2 bytes. Among them, low 12 bits are effectively used, and reserved high 4 bits are not used. To 80 bands image data file, the file size is 1024 pixels $* 1024$ pixels* 2 Byte $* 80$ bands, that is 167772160Byte.

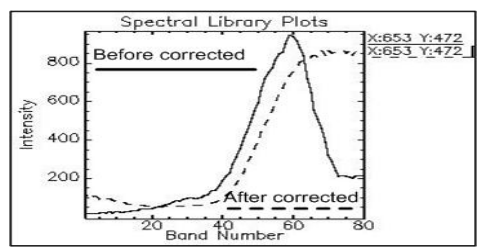

FIGURE III. DATA PREPROCESSING.

Affected by various factors in the light path, radiation correction of original data must first be performed [7]. In reference 7 , radiation correction algorithm was introduced in detail. Figure 3 shows the optic spectrum curve before and after the radiation correction. The solid line is the spectrum curve before correction, and dotted line is the spectral curve after calibration. The corresponding band of spectral curve is 1 band $\sim 80$ band.

\section{Outer Nuclear Layer Thickness Calculation}

Retinal thickness of DR rats will be reduced as the disease worsens, especially the ONL thickness decreases seriously [8]. The following a series of formulas about how to calculate the ONL thickness are proposed. Figure 4 is the optic nerve image of E2 group rats in $627.1 \mathrm{~nm}$, and the image had been pretreated by radiometric correction in spatial dimension and spectral dimension. From this image we seek calculation method of the ONL thickness.

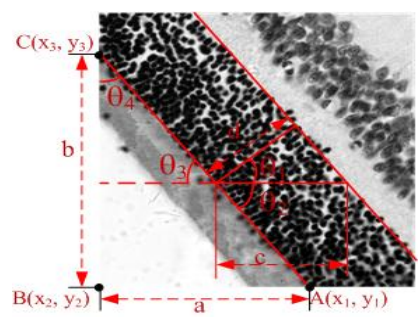

FIGURE IV. THE ONL THICKNESS CALCULATION.
From Figure 4, coordinates of A, B, C can be obtained, then

$$
\begin{aligned}
& \because a=x_{1}-x_{2}, b=y_{2}-y_{3} \therefore \operatorname{tg} \theta_{4}=\frac{a}{b} \\
& \because \quad \theta_{1}+\theta_{2}=90^{\circ}, \theta_{3}+\theta_{4}=90^{\circ}, \theta_{3}=\theta_{2} \\
& \therefore \quad \theta_{1}=\theta_{4}
\end{aligned}
$$

Because the length of $C$ is known, then

$d=c \cdot \cos \theta_{1}=c \cdot \cos \theta_{4}$

By N samples, $d_{i}$ ( $i$ from 1 to $N$ ) is obtained respectively and then averaging.

$$
d_{\text {avg }}=\frac{1}{N} \sum_{i=1}^{N} d_{i}, \quad \mathrm{i}=1,2, \ldots . \mathrm{N}
$$

\section{E. SAM and Correlation Coefficient}

SA (Spectral Angle) refers to the angle formed by the two pixel vector having the same wavelength range in the spectral space. It can be used as an important indicator of the measure of spectral similarity. It is defined as follows:

$$
\cos (\vec{x}, \vec{y})=\frac{\vec{x}^{T} \vec{y}}{\|\vec{x}\| \cdot\|\vec{y}\|}=\frac{\vec{x}^{T} \vec{y}}{\sqrt{\left(\vec{x}^{T} \vec{x}\right)\left(\vec{y}^{T} \vec{y}\right)}}
$$

The angle between the two spectra is smaller, the two spectra are more similar. Spectral angle is expressed by cosine value, which means that the cosine value is greater closely to 1 , spectrum comparability is higher.

Spectrum comparability between the two spectra can also be expressed by correlation coefficient. It is defined as follows:

$$
\gamma(\vec{x}, \vec{y})=\frac{(\vec{x}-\bar{x})^{T}(\vec{y}-\bar{y})}{\sqrt{\left[(\vec{x}-\bar{x})^{T}(\vec{x}-\bar{x})(\vec{y}-\bar{y})^{T}(\vec{y}-\bar{y})\right]^{2}}}
$$

$\bar{x}, \bar{y}$ are the average of two vectors. By formula (3), it indicates that greater is the correlation coefficient, higher is the similarity between the two spectra.

\section{EXPERIMENTAL RESULTS}

The following quantitative analysis comes from the four aspects: the thickness of the outer nuclear layer, comparing the relative error of the spectrum, spectral similarity comparison, and optic nerve density of ONL, which illustrate AAV2-EPO has an important role of treating DR rats. 
A. Comparison of Outer Nuclear Layer Thickness

TABLE I. COMPARISON OF THE ONL THICKNESS OF N, D, E1, E2, E3 GROUPS.

\begin{tabular}{l|llll}
\hline Group & ROI & $C$ (pixel) & $d$ (pixel) & AVG \\
\hline \multirow{2}{*}{$\mathrm{N}$} & 1 & 236 & 228 & \\
& 2 & 232 & 224 & 228 \\
\hline \multirow{3}{*}{ E1 } & 3 & 239 & 231 & \\
& 1 & 263 & 263 & 256 \\
\hline \multirow{3}{*}{ E2 } & 2 & 250 & 250 & \\
& 3 & 256 & 256 & 354 \\
\hline \multirow{3}{*}{ E3 } & 1 & 474 & 352 & \\
& 2 & 486 & 361 & \\
\hline \multirow{3}{*}{ D } & 3 & 469 & 349 & 116 \\
& 1 & 139 & 89 & \\
\hline
\end{tabular}

Based on the ONL thickness calculation, the ONL thickness of N, D, E1, E2, E3 groups were respectively calculated according to the formula (1). The results are shown in Table 1. By the average of the ONL thickness of three samples, the result is $d_{E 2}>d_{E 1}>d_{N}>d_{D}>d_{E 3}$. This indicates that retinal ONL thickness of DR rats is changed after different doses of AAV2-EPO treatment. The most obvious increase in retinal ONL thickness belong to E2 group, followed by the E1 group, but retinal ONL thickness of E3 group is lower than the normal group. In a series of experiments, the AAV2-EPO has a good effect to increase retinal ONL thickness, but the drug dose should be controlled in the appropriate range. The results show that the medium dose of AAV2-EPO has a significant effect to increase the retina ONL thickness of DR rats.

\section{B. Comparison of the Relative Error of the Spectrum}

For a more intuitive comparison of the spectral characteristics of $\mathrm{N}$ group, $\mathrm{D}$ group and $\mathrm{E} 1, \mathrm{E} 2$, and $\mathrm{E} 3$ treatment group in order to further assess AAV2-EPO efficacy of the different doses to promoting cells performance, E1, E2, and $\mathrm{E} 3$, and $\mathrm{D}$ groups are compared respectively with $\mathrm{N}$ group. The relative errors between them are calculated. The relative error results are shown in Figure 5.

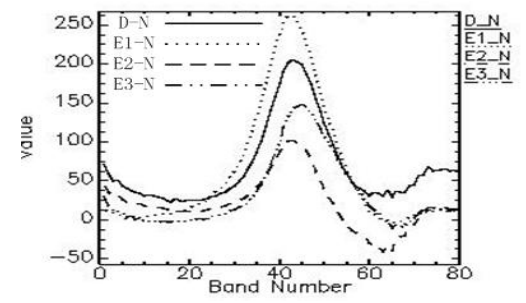

FIGURE V. RELATIVE ERROR CURVE OF D\&N, E1\&N, E2\&N, E3\&N.

By analyzing Figure8, the relative error curves were tested characteristics of a single peak. Relative errors of E2 \& N, E3 $\& N$ are less than that of $D \& N$, but relative error of $E 1 \& N$ is slightly larger than the that of D \& N. Compared with these error curves, the peak of error curve about E2 \& N is smallest, and the curve is closest to the horizontal line with 0 value, which also shows the spectral characteristics of the E2 group cells are most similar with $\mathrm{N}$ group. It also exemplifies that the medium dose treatment with AAV2-EPO to DR rats has optimal efficacy. After the medium dose treatment the biochemical properties of retinal ONL cells are closer to normal cells.

\section{Spectral Similarity Comparison on the Spectrum Angle and the Correlation Coefficient}

In order to better compare efficacy of different dose AAV2of EPO to DR rats, the experiment was divided into 3 times, and the spectral angle and the correlation coefficient of D \& N, E1 \& N, E2 \& N, E3 \& N were respectively calculated according to the formula (2) and (3), and then calculate the average. Table 2 gives comparative statistic on the spectral similarity about D \& N, E1 \& N, E2 \& N, E3 \& N.

TABLE II. COMPARATIVE STATISTICS ON THE SPECTRAL SIMILARITY ABOUT D\&N, E1\&N, E2\&N, E3\&N.

\begin{tabular}{l|l|l|l}
\hline & samples & $S A M(\cos \theta)$ & $\begin{array}{l}\text { Correlation } \\
\text { coefficient }\end{array}$ \\
\hline \multirow{5}{*}{1} & D\& N & 0.99595628 & 0.99250456 \\
& E1\&N & 0.99367216 & 0.98705755 \\
& E2\&N & 0.99679765 & 0.99262367 \\
& E3\&N & 0.99735509 & 0.99541519 \\
\hline \multirow{5}{*}{2} & D\&N & 0.99370479 & 0.99047270 \\
& E1\&N & 0.99026969 & 0.98224705 \\
& E2\&N & 0.99756444 & 0.99552098 \\
& E3\&N & 0.99565674 & 0.99285093 \\
\hline \multirow{5}{*}{3} & D\& N & 0.98944676 & 0.98643765 \\
& E1\&N & 0.98266539 & 0.97425538 \\
& E2\&N & 0.99654906 & 0.99503359 \\
& E3\&N & 0.99471248 & 0.99092265 \\
\hline \multirow{3}{*}{ AVG } & D\& N & 0.99379086 & 0.99083748 \\
& E1\&N & 0.99008039 & 0.98292460 \\
& E2\&N & 0.99748064 & 0.99515430 \\
\hline \multirow{6}{*}{ FrOM } & E3\&N & 0.99615270 & 0.99347978 \\
\hline
\end{tabular}

From the experimental data of Table II, it can be seen that after treatment by AAV2-EPO, spectral angle and spectral correlation coefficients of E1 \& $\mathrm{N}$ group are substantially smaller than $\mathrm{D} \& \mathrm{~N}$ group. The spectral angle and spectral correlation coefficients of E2 \& N, E3 \& N groups are substantially all greater than D \& N group, especially E2 \& N group has maximum spectral angle and correlation coefficient. It further demonstrates that the biochemical properties of ONL cells of DR rats are closer to normal cells after using AAV2EPO treatment, especially medium dose treatment of AAV2EPO has the most obvious effects.

\section{CONCLUSION}

As mentioned above, molecular hyperspectral image data of rat retinal tissue sections were obtained in the experiment, and respectively divided into normal group $\mathrm{N}$, diabetic group $\mathrm{D}$ and AAV2-EPO drug treatment group (including low doses E1 group, middle doses E2 group, high dose E3 group). The quantitative analysis from three aspects have illustrated that AAV2-EPO has a certain effect to DR rat, and especially medium dose treatment of AAV2-EPO has the most obvious effects.

\section{ACKNOWLEDGMENT}

This work is supported in part by the National Natural Science Foundation of China (Grant No. 61240006, 61177011). 


\section{REFERENCES}

[1] Danis RP, Davis MD. Proliferative Diabetic Retinopathy [M]. Contemporary Diabetes, 2008, 1: 29-65.

[2] Zhang Jingfa, Wu Yalan, Jin Ying, et al. Intravitreal Injection of Erythropoietin Protects both Retinal Vascular and Neuronal Cells in Early Diabetes [J]. Investigative Ophthalmology \& Visual Science, 2008, 49(2): 732-742.

[3] ZY Wang, KK Zhao, PQ Zhao, et al. Erythropoietin therapy for early diabetic retinopathy through its protective effects on retinal pericytes $[\mathrm{J}]$. Medical Hypotheses, 2011, 76(2): 266-268.

[4] LI QingLi, XUE YongQi, ZHANG JingFa et al. Microscopic hyperspectral imaging studies of normal and diabetic retina of rats [J]. Science in China Series C: Life Sciences, 2008, 51(9): 789-794.

[5] K Stieger, G Le Meur, F Lasne, et al. Long-term doxycycline-regulated transgene expression in the retina of nonhuman primates following subretinal injection of recombinant AAV vectors [J]. Molecular Therapy, 2006, 13:967-975.

[6] M Schmidt, A Voutetakis, S Afione, et al. Adeno-associated virus type 12 (AAV12): a novel AAV serotype with sialic acid-and heparan sulfate proteoglycan-independent transduction activity [J]. Journal of Virology, 2008, 83(3): 1399-1406.

[7] HY Liu, QL Li, B Gu, et al. Performance Analysis And Radiometric Correction of Novel Molecular Hyperspectral Imaging System [J]. Spectroscopy and Spectral Analysis, 2012, 32(11): 3161-3166.

[8] Q Wang, S Gorbey, F Pfister, et al. Long-term treatment with suberythropoietic Epo is vaso-and neuroprotective in experimental diabetic retinopathy $[\mathrm{J}]$. Cellular Physiology and Biochemistry, 2011, 27(6):769-782. 